\title{
Epigenetic therapy gains momentum
}

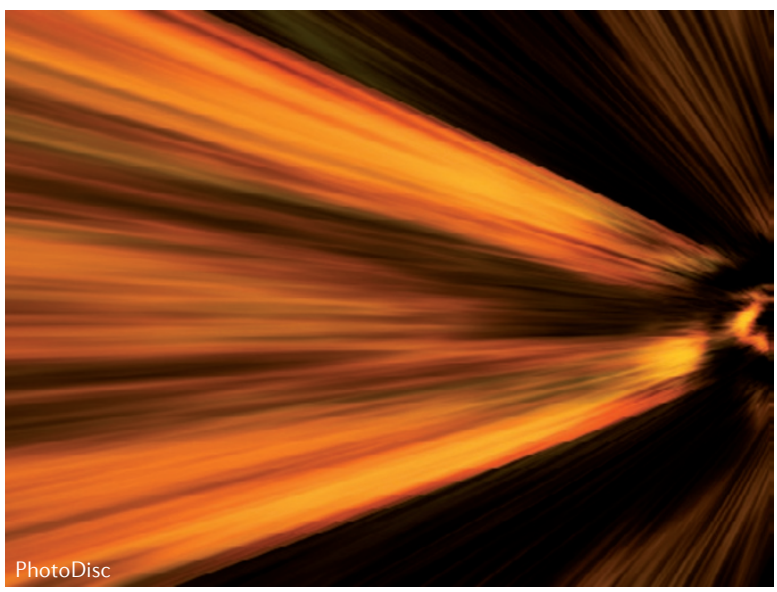

Trimethylation of histone $\mathrm{H} 3$ on lysine 27 (H3K27me3) represses gene transcription; this epigenetic modification is catalysed by the polycomb repressive complex 2 (PRC2), of which the enzymatic subunit is either EZH1 or EZH2. Both activation and loss of EZH2 methyltransferase activity have been described in different cancer types; in diffuse large B cell lymphoma (DLBCL), activating point mutations are common. It is unknown whether

inhibited growth ... of DLBCL cell lines with EZH2activating mutations is highly selective for EZH2 over other methyltransferases, including EZH1. GSK126 induced a reduction of H3K27me3 in DLBCL cell lines with both wild-type and mutant EZH2. Testing the compound in a panel of cell lines of different B cell lymphoma subtypes revealed that GSK126 most effectively inhibited the growth of DLBCL cells with the activating mutations Y641 or A677. This occurred through both cytostatic and cytotoxic responses, and was recapitulated by short hairpin RNAs (shRNAs) against EZH2. GSK126 and EZH2 shRNAs also led to transcriptional changes - primarily derepression of $\mathrm{H} 3 \mathrm{~K} 27 \mathrm{me} 3$ targets - in DLBCL cell lines that were sensitive to GSK126. Interestingly, a muted transcriptional response and lower global H3K27me3 levels were observed in cell lines with EZH2activating mutations that were less sensitive to GSK126, indicating that H3K27me3 levels combined with an EZH2-activating mutation might better predict response. GSK126 also inhibited the growth of established subcutaneous xenografts of two EZH2-mutant DLBCL cell lines, and significantly improved survival of the mice. Minimal side effects were observed in mice carrying xenograft tumours, and in immunocompetent mice, which is important given the roles of EZH2 in haematopoiesis and identification of loss-of-function
EZH2 mutations in myeloid malignancies. Other compounds in the same chemical series as GSK126 are described by Verma et al.

Knutson et al. identified another SAM-competitive and selective inhibitor of EZH2, EPZ005687, which has not yet been optimized for possible therapeutic use. In vitro assays indicated that EPZ005687 directly inhibits enzymatic activity of EZH2 rather than PRC2 complex formation. Further testing in DLBCL cell lines showed that EPZ005687 blocked H3K27me3 regardless of EZH2 mutation status, but only inhibited growth (through both cytostatic and cytotoxic effects) of DLBCL cell lines with EZH2activating mutations. These authors also conducted gene expression analysis in EPZ005687-treated EZH2-mutant cells and found that inhibition of EZH2 derepressed known EZH2 targets.

It will be interesting to see whether the potential of EZH2 inhibitors is realized in clinical testing.

Sarah Seton-Rogers

ORIGINAL RESEARCH PAPERS

Knutson, S. K. et al. A selective inhibitor of EZH2 blocks H3K27 methylation and kills mutant lymphoma cells. Nature Chem. Biol. 8, 890-896 (2012) | McCabe, M. T. et al. EZH2 inhibition as a therapeutic strategy for lymphoma with EZH2activating mutations. Nature 10 Oct 2012 (doi:10.1038/nature11606) |Verma, S. K. et al. Identification of potent, selective, cell-active inhibitors of the histone lysine methyltransferase EZH2. ACS Med. Chem. Lett. 19 Oct 2012 (doi:10.1021/ml3003346) 